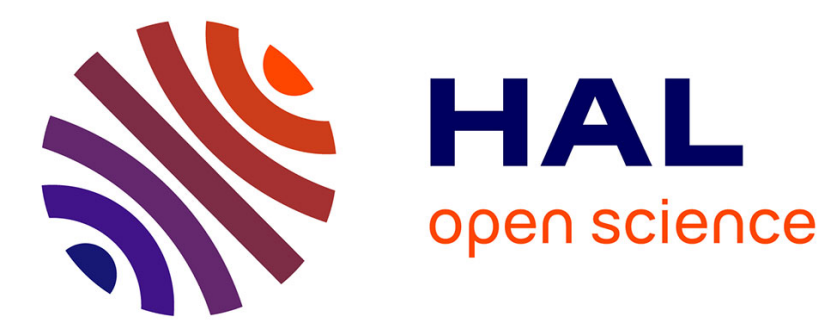

\title{
The energy spread of some negative ions from a sputter source
}

\author{
G. Doucas
}

\section{To cite this version:}

G. Doucas. The energy spread of some negative ions from a sputter source. Revue de Physique Appliquée, 1977, 12 (10), pp.1465-1469. 10.1051/rphysap:0197700120100146500 . jpa-00244349

HAL Id: jpa-00244349 https://hal.science/jpa-00244349

Submitted on 1 Jan 1977

HAL is a multi-disciplinary open access archive for the deposit and dissemination of scientific research documents, whether they are published or not. The documents may come from teaching and research institutions in France or abroad, or from public or private research centers.
L'archive ouverte pluridisciplinaire HAL, est destinée au dépôt et à la diffusion de documents scientifiques de niveau recherche, publiés ou non, émanant des établissements d'enseignement et de recherche français ou étrangers, des laboratoires publics ou privés. 


\title{
THE ENERGY SPREAD OF SOME NEGATIVE IONS FROM A SPUTTER SOURCE
}

\author{
G. DOUCAS \\ Nuclear Physics Laboratory, University of Oxford, Oxford, England
}

\begin{abstract}
Résumé. - Des mesures de la distribution d'énergie sur des faisceaux de ${ }^{12} \mathrm{C}^{-}, 32 \mathrm{~S}^{-}, 127 \mathrm{I}^{-}, \mathrm{H}^{-}$et $16 \mathrm{O}^{-}$d'énergie $20 \mathrm{keV}$ n'ont montré aucunes différences évidentes entre $\mathrm{H}^{-}$et $16 \mathrm{O}^{-}$d'une part (on pense qu'ils sont produits dans une couche absorbée de la cible) et ${ }^{12} \mathrm{C}^{-},{ }^{32} \mathrm{~S}^{-}$et ${ }^{127 \mathrm{I}^{-}}$d'autre part, qui font partie du matériau de la cible. Tous les spectres ont un seul pic et une largeur de l'ordre de 10$12 \mathrm{eV}$. La signification de la distribution en énergie dans le calcul de la brillance du faisceau est également discutée.
\end{abstract}

\begin{abstract}
Energy distribution measurements on $20 \mathrm{keV}$ beams of ${ }^{12} \mathrm{C}^{-},{ }^{32} \mathrm{~S}^{-},{ }^{127} \mathrm{I}^{-}, \mathrm{H}^{-}$and ${ }^{16} \mathrm{O}^{-}$ have shown no obvious differences between $\mathrm{H}^{-}$and ${ }^{16} \mathrm{O}^{-}$on the one hand, which are believed to be produced in an adsorbed layer on the target, and ${ }^{12} \mathrm{C}^{-},{ }^{32} \mathrm{~S}^{-}$and ${ }^{127} \mathrm{I}^{-}$on the other which are part of the target material. All spectra have a single peak and a FWHM of the order of 10-12 eV. The significance of the energy distribution in the calculation of beam brightness is also discussed.
\end{abstract}

1. Introduction. - Since it was first introduced in 1972 [1], the sputter source has attracted considerable attention, both because of its simplicity and its versatility in producing a wide variety of heavy negative ions. Most of the work until now has concentrated on the production of a wider variety of negative ions [2], on increasing the intensity of the negative beam $[3,4]$ and, finally, on the measurement of the quality of the beam [5]. While both variety and intensity have improved, the quality of the beam, expressed as emittance or brightness, continues to remain relatively modest, especially when compared to the brightness of a beam derived from a direct extraction duoplasmatron source.

The purpose of the present work was to investigate the fundamental ion production process of this type of source, i.e. sputtering, through the study of the energy distribution of the negative particles. Since, in the absence of non-conservative forces, the emittance of the beam is determined once and for all at the origin of the ions, this type of measurement is expected to provide a useful insight into the factors that give rise to the relatively large 'emittance of the sputtered beams. From the surface physics point of view these measurements are also of some interest because there are few papers on the energy spread of negative ions $[6,7]$ and, to the author's knowledge, only one on negative ions derived from a surface covered by a layer of an alkaline element [8].

All the energy spectra were obtained by means of a retarding field energy analyzer.
2. Experimental. - The ion source used in these experiments is a Middleton-type sputter source (Fig. 1) and has been described in the literature [3]

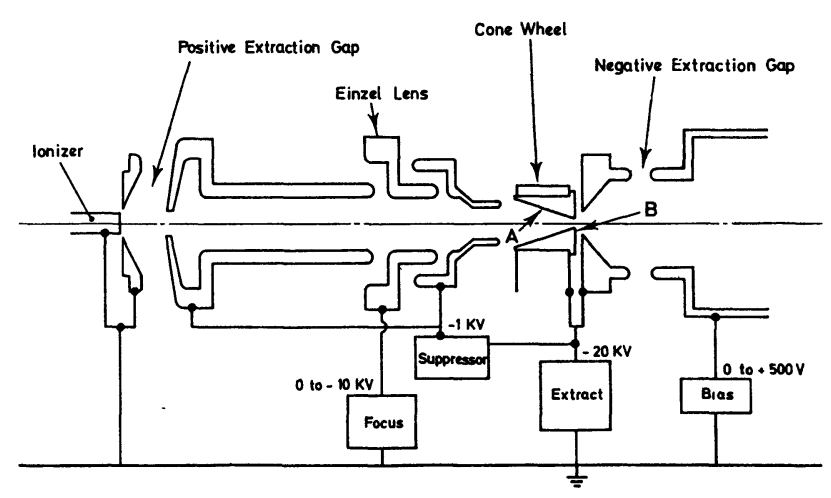

FIG. 1. - Schematic arrangement of the ion source.

Briefly, a fairly intense (up to $5 \mathrm{~mA}$ ) positive caesium beam is generated by surface ionization on a hot $\left(\sim 1200^{\circ} \mathrm{C}\right.$ ) porous tungsten surface. This beam is then focussed and allowed to bombard, with a typical energy of $20 \mathrm{keV}$, a solid target of hollow conical shape. The sputtered negative ions are extracted through a $1 \mathrm{~mm}$ diameter hole at the apex of the cone by a potential of $20 \mathrm{kV}$ plus a small additional bias of a few hundred volts, which has a significant effect on the negative beam intensity. The caesium plays a dual role : it acts as a sputtering agent and it also enhances the production of negative ions $[9,10]$.

The layout of our experimental arrangement is 
shown in figure 2 . The negative beam is focussed by a gridded lens and mass analyzed by a $30^{\circ}$ magnet. The image is formed, approximately, at the plane of two pairs (one horizontal, one vertical) of movable slits. This set of slits is followed by a removable Faraday cup, the retarding field energy analyzer and a second Faraday cup. The slits are adjusted on the optical axis of the system so as to select a small element of the beam, usually $1 \times 1 \mathrm{~mm}^{2}$. The source and focussing conditions are then adjusted in order to maximise the current at the first cup. This is then removed from the beam path and the beam is allowed to drift through the analyser. The beam current that can surmount the potential barrier of the analyzer is measured by means of the second cup.

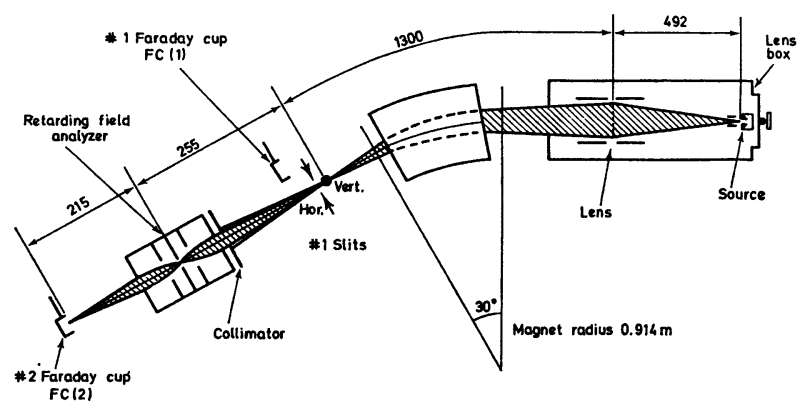

FIG. 2. - Layout of experimental arrangement.

The retarding field energy analyzer is similar to that described by Simpson [7]. It consists of two immersion lenses placed back to back and is shown schematically in figure 3 . The first lens retards the beam and focusses it through a $1 \mathrm{~mm}$ aperture on the retarding electrode; the second lens accelerates the beam emerging from this aperture and focusses it again further downstream, approximately at the position of the second Faraday cup. The whole system is symmetric (i.e. the two half-lenses are identical) although this is not essential. The electrical connections are also shown on figure 3 . The central electrode with the $1 \mathrm{~mm}$ aperture carries the extract potential of the negative ions plus the small variable bias voltage;

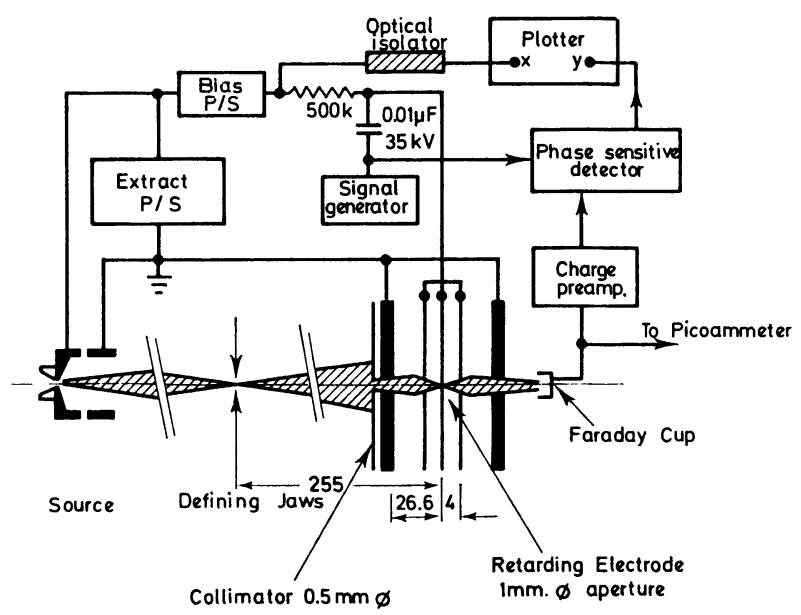

FIG. 3. - Schematic of energy analyzer and electronics. these two voltages form the potential barrier that the negative ions have to overcome. Hence, this electrode is referred to as the retarding electrode. The two intermediate electrodes adjacent to it are also connected to it electrically. This ensures that, for our specific geometry, an object placed approximately at infinity (i.e. the adjustable slits) is brought to a focus on the retarding plane. The two outer electrodes are at earth potential.

If the particles in the beam have a velocity distribution $f(v)$, then the current density $J$ through the aperture in the retarding plane of the analyzer, is given by the expression

$$
J=e \int_{\nu_{0}}^{\infty} v f(v) \mathrm{d} v
$$

where $v_{\mathrm{o}}$ is the lowest velocity collected by the analyzer.

Since $v \mathrm{~d} v=\frac{e}{m} \mathrm{~d} V$ the above expression can be written

$$
J=\frac{e^{2}}{m} \int_{v_{0}}^{\infty} f(v) \mathrm{d} V
$$

Thus an $I$ vs. $V$ curve would be an integral curve which, after differentiation, yields the velocity (or momentum) distribution in a direction perpendicular to the equipotential lines at the barrier and not the total energy. The difference between this normal energy and the total energy of the particle is the resolution of the analyzer. This resolution $\frac{\Delta E}{E}$ has been shown to be approximately equal to $9.5 \times 10^{-4}$ for our analyzer and experimental conditions [12]. Thus, for a $20 \mathrm{keV}$ beam, the maximum possible error is $\Delta E \simeq 19 \mathrm{eV}$.

The differentiation of the $I v s$. $V$ curve was done electronically as shown in figure 3 . The signal generator produces a sinusoidal modulating signal of approximately $1 \mathrm{~V}$ peak-to-peak at $230 \mathrm{~Hz}$. This signal is superimposed, via a $0.01 \mu \mathrm{F}, 35 \mathrm{kV}$ capacitor, onto the DC bias voltage that is applied to the retarding electrode of the analyzer; the same signal is also fed into a phase sensitive detector at ground potential. The other input to this detector is the output of the charge preamplifier which is used to amplify the current collected on the second Faraday cup (FC2). The output of the phase sensitive detector is the required derivative $\frac{\mathrm{d} I}{\mathrm{~d} V}$.

The signal-to-noise ratio deteriorates with increasing retarding potential and at the end of the scan it drops to $10: 1$ or, possibly, slightly worse. The system is capable of higher gain on the phase-sensitive detector but this would be at the expense of longer time constants, i.e. slower scanning speed for the retarding potential. As a compromise, a time constant of $0.22 \mathrm{~s}$ was chosen and the scanning speed was adjusted to $30 \mathrm{~V} / \mathrm{min}$. which was slow enough to prevent hysteresis. The value of $230 \mathrm{~Hz}$ (or occasionally $860 \mathrm{~Hz}$ ) for the modulation frequency was chosen because it is 
clear of the $50 \mathrm{~Hz}$ harmonics and it produces the minimum amount of noise; the amplitude of the signal ( $1 \mathrm{~V}$ peak-to-peak) was found to be adequate for a reasonable output from the detector, without introducing any significant distortion of the spectrum.

3. Discussion. - Energy distributions were obtained for negative ions of three types :

(a) particles sputtered from an elemental cone, e.g. ${ }^{12} \mathrm{C}^{-}$from graphite,

(b) particles sputtered from a cone made of a chemical compound, e.g. ${ }^{32} \mathrm{~S}^{-}$from $\mathrm{PbS},{ }^{127 \mathrm{I}^{-}}$from $\mathrm{NaI}$,

(c) particles sputtered or desorbed from a surface adsorbate e.g. $\mathrm{H}^{-}$from $\mathrm{NH}_{3}$ on $\mathrm{Ca},{ }^{16} \mathrm{O}^{-}$from $\mathrm{O}_{2}$ on Li.

In all cases, the energy of the negative beam was $20 \mathrm{keV}$.

The corrections that have to be made to the energy spectra in order to take account of some systematic errors have been discussed in detail in ref. [12]. Figure 4 shows three typical energy distributions for ${ }^{12} \mathrm{C}^{-},{ }^{32} \mathrm{~S}^{-}$and ${ }^{127 \mathrm{I}^{-}}$ions (curves $\mathrm{a}, \mathrm{b}$ and $\mathrm{c}$, respectively), after the appropriate corrections. The following general comments can be made about these distribu-
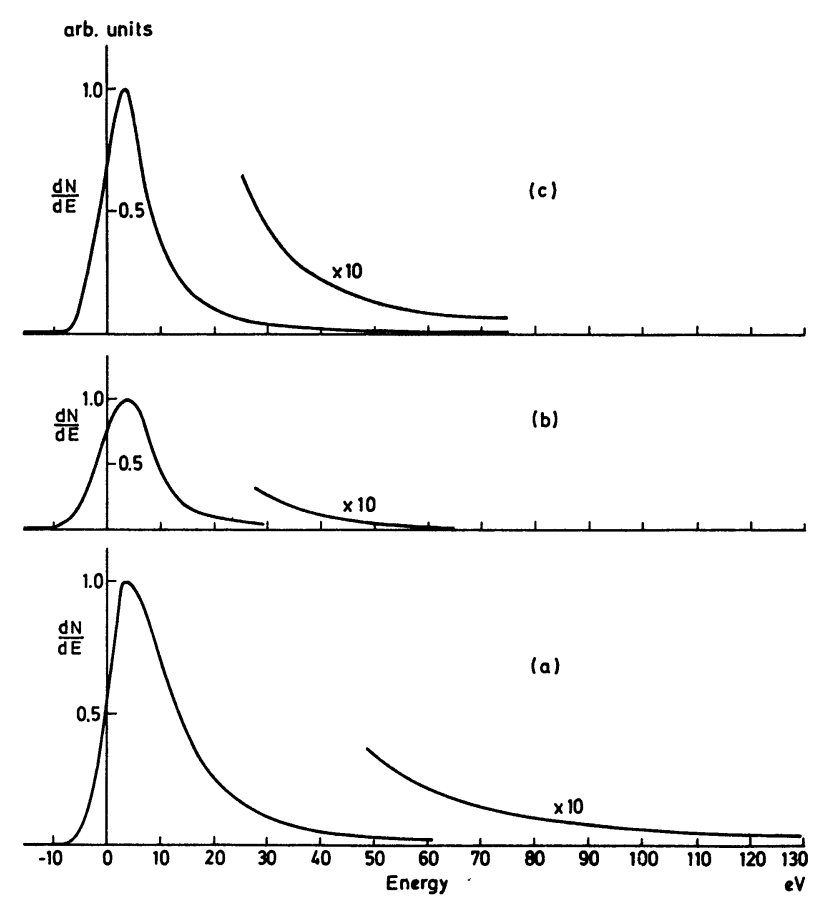

FIG. 4. - Typical energy distribution curves for three different negative ions at $20 \mathrm{keV}$.
(a) ${ }^{12} \mathrm{C}^{-}$
(b) ${ }^{32} \mathrm{~S}^{-}$
(c) $127 \mathrm{I}^{-}$

tions and, similarly, about all the distributions reported in this paper :

(a) The general appearance of the spectrum is very similar to that of the sputtered neutrals; its most obvious characteristic is the high energy tail which decays with energy according to a law $\frac{\mathrm{d} N}{\mathrm{~d} E} \infty \mathrm{E}^{\sim n}$ with $n \simeq 2$. This is shown more clearly in figure 5 where the same curves have been plotted on a log-log graph. The lines are a least-squares fit to a weighted set of data points and the error bars on line (a) should be considered as typical. It can be seen that the value of $n=2.16$ for the $127 \mathrm{I}^{-}$beam is close to the theoretically predicted value of 2 for sputtered neutrals while $n=2.29$ for the ${ }^{12} \mathrm{C}^{-}$beam, again in reasonable agreement with neutral sputtering theory. For the $32 \mathrm{~S}^{-}$beam $n=3.27$ and the agreement is poor, although values as low as 2.3 (and as high as 4.3 ) have been recorded for various $32 \mathrm{~S}^{-}$spectra.

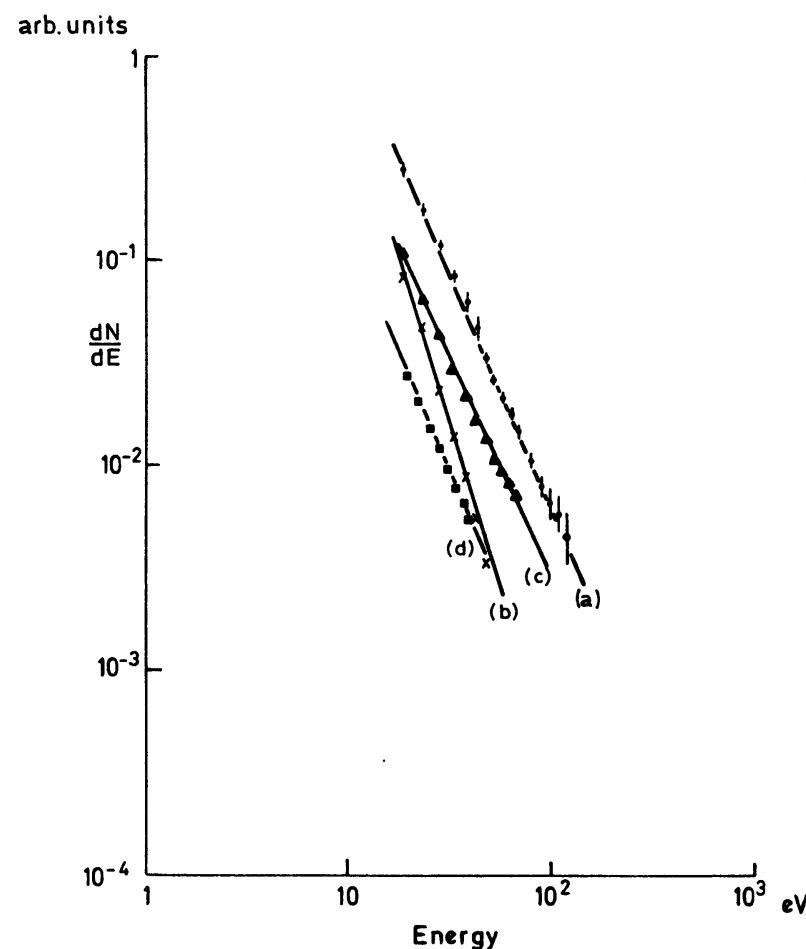

Fig. 5. - Calculation of power $n$ in the relationship $\frac{\mathrm{d} N}{\mathrm{~d} E} \propto E^{-n}$ for
four different ions. (a) ${ }^{12} \mathrm{C}^{-}, n=2.29$ (b) ${ }^{32} \mathrm{~S}^{-}, n=3.27$ (c) $127 \mathrm{I}^{-}, n=2.16$ (d) $\mathrm{H}^{-}, n=2.21$.

(b) The peak of the distribution occurs at a few $\mathrm{eV}$ but its exact position is uncertain because of two factors, i) the convolution of the true distribution function with the resolution function of the instrument to provide the measured distribution function and ii) the possible effect of a difference in work functions between the ion emitting surface and the retarding electrode.

(c) The full width at half maximum (FWHM) of the distribution is quite small, of the order of 10$12 \mathrm{eV}$.

(d) On a number of occasions, a different type of spectrum has been observed for ${ }^{12} \mathrm{C}^{-}$and ${ }^{32} \mathrm{~S}^{-}$ion beams. This is much broader, of the order of $70-80 \mathrm{eV}$ and has two peaks, either clearly resolved or merged 
to form a rather flat plateau. The origin of this energy distribution, is not as yet clearly understood.

The energy spectra of negative ions that are usually derived by gas injection, like $\mathrm{H}^{-}$and ${ }^{16} \mathrm{O}^{-}$, are very similar to the ones shown in figure $4 . \mathrm{H}^{-}$was produced by $\mathrm{NH}_{3}$ injection on a Ca cone and the mean FWHM of a number of measurements was $7.6 \mathrm{eV}$ while the position of the peak occured at $1.8 \mathrm{eV}$. The ${ }^{16} \mathrm{O}^{-}$ion was produced by oxygen injection onto a $\mathrm{Ti}$ cone or, alternatively, onto a $\mathrm{Li}$ cone. The mean values for peak position and FWHM were $4.8 \mathrm{eV}$ and $13.1 \mathrm{eV}$ respectively. It is worth noting that the $\mathrm{H}^{-}$ion appears, consistently, to have an energy distribution somewhat narrower than the other ions studied in these experiments. The value of $n$ for $\mathrm{H}^{-}$is 2.2 (line $\mathrm{d}$ in Fig. 5).

It might be useful at this point to relate, at least quantitatively, the energy distribution of the sputtered ions, which is a parameter describing a physical process, to the concept of emittance, which is derived from statistical mechanics and is used to describe the collective behaviour of an ensemble of particles. The fundamental assumption here is that the particles, on leaving the source, are only subjected to conservative forces. Thus, the emittance is determined only by the physical processes that occur at the point of origin.

The emittance is defined as the volume occupied by the particles of the beam in the six-dimensional phase space made up from the three position coordinates $x$, $y, z$ and the corresponding momentum coordinates $p_{x}, p_{y}$ and $p_{z}$. If the beam is to have a small emittance then it is a necessary condition that its extent in either the real space or the momentum space is small. If one now looks at the point of origin of the ions, the sputtered surface in our case, it is obvious that the extent in momentum space is determined by the angular and energy (or momentum) distributions of the sputtered particles. Thus, if these two distributions are known, as well as the spatial distribution of the ions on the emitting surface, it is possible, in principle, to calculate the brightness of the source. A particularly simple example is demonstrated in the appendix. A rather crude, order of magnitude calculation shows that since the maximum error of the analyzer at $20 \mathrm{keV}$ is approximately $20 \mathrm{eV} \mathrm{[12]} \mathrm{and} \mathrm{since} \mathrm{this}$ error represents the transverse energy (or momentum) of the particle, then the divergence of the beam is approximately $r^{\prime}=\sqrt{\frac{20}{2 \times 10^{4}}} \simeq 32 \mathrm{mrad}$. If the source radius is of the order of $1 \mathrm{~mm}$, then the twodimensional emittance is $4.5 \mathrm{~mm} \cdot \operatorname{mrad}(\mathrm{MeV})^{1 / 2}$. This is of the correct order of magnitude.

In many cases the emittance of a beam is expressed as a four- (or sometimes two-) dimensional volume, instead of the full six dimensional volume. The reason for this is that the $z$-coordinate has no significance, unless the beam possesses time structure and the $p_{z}$ coordinate represents a fractional change to the beam's overall momentum. Hence the $\left(z, p_{z}\right)$ space is often omitted. If, however, the beam does possess time structure, then the $\left(z, p_{z}\right)$ space is useful and is usually expressed as a $(t, \Delta E)$ space, where $t$ is the time spread of the beam and $\Delta E$ its fractional energy spread. It is obvious that, in such a case, knowledge of the energy spread of the beam is essential before its bunching properties can be determined.

4. Summary and Conclusions. - The energy distribution of a variety of negative ions produced in a Middleton-type sputter source has been found to resemble that of sputtered neutrals : the energy spectra are asymmectric, with a high energy tail decaying with energy as $E^{-n}$, where $n$ is usually, but not always, approximately equal to 2 . The full-width at half maximum (FWHM) of the distribution is of the order of 10-12 eV, for beam energies of $20 \mathrm{keV}$. There is no appreciable difference between the spectra of ions like $\mathrm{H}^{-}$and ${ }^{16} \mathrm{O}^{-}$, which are produced in the adsorbed layer on the target and ions like ${ }^{12} \mathrm{C}^{-}, 32 \mathrm{~S}^{-}$ and $127 \mathrm{I}^{-}$which originate from the target material itself.

A knowledge of the energy distribution of the negative particles allows, in principle, the calculation of the source brightness provided that the angular and spatial distributions are also known.

Finally, in view of the rather small energy spreads that have been observed, it is reasonable to assume that the relatively large emittance of this type of source is most probably due to a large emitting area and/or to a large angular distribution of the sputtered particles.

Acknowledgements. - The author in indebted to Mr. H. R. McK. Hyder for a number of discussions on energy spreads, to Mr. A. B. Knox for assistance in the measurements and to Mr. P. Barratt for the installation and maintenance of the electronic differentiation system.

The work has been supported financially by the Science Research Council.

Appendix. - The following demonstrates the possibility of calculating source brightness $B$ once the energy, angular and spatial distributions are known. A number of simplifying assumptions have been made :

1. There is complete independence of the momentum coordinates from the position coordinates; there is also rotational symmetry in both real and momentum spaces.

2. In real space, the source is a circle of radius $r_{0}$, emitting ions with a uniform density $\rho_{\mathrm{o}}$ particles $/ \mathrm{m}^{2}$.

3. The momentum space is expressed in terms of total momentum $p$ and the two angles $\varphi, \theta$. If the particles are emitted with a cosine angular distribution and because of the rotational symmetry, the density of points in this space is given by

$$
P(p, \varphi)=a P_{1}(p) \cos \varphi
$$


where $a$ is a constant expressed in particles/ $[p]^{2}$ and $P_{1}(p)$ is the measured momentrum (or energy) distribution. The symbol $[p]$ is used to denote momentum coordinates $\left(\mathrm{kg} \cdot \mathrm{ms}^{-1}\right)$.

The current emitted by the surface can be written down in the form

$$
I=e N \quad N(\text { particles } / \mathrm{s} .)
$$

but, because of assumption (1),

$N=4 \pi^{2} \iint \rho(r) r \mathrm{~d} r \iint a P_{1}(p) \cos \varphi p^{2} \sin \varphi \mathrm{d} p \mathrm{~d} \varphi$

and on the basis of assumption (2),

$$
N=2 \pi^{2} \rho_{0} r_{0}^{2} a \int_{0}^{\infty} P_{1}(p) p^{2} \mathrm{~d} p \int_{0}^{\frac{\pi}{2}} \cos \varphi \sin \varphi \mathrm{d} \varphi
$$

or $\quad N=\pi^{2} \rho_{0} a r_{0}^{2} \int_{0}^{\infty} P_{1}(p) p^{2} \mathrm{~d} p$
At this point one should substitute into (2) the analytical expression for the momentum (or energy) distribution that has been determined experimentally. Let us assume, for ease of calculation, that $P_{1}(p)$ is a $\delta$-function at $p=p_{\mathrm{o}}$. This means that in momentum space all the particles are on the surface of a spherical shell with radius $p_{0}$. Eq. (2) then becomes

$$
\begin{aligned}
& N=\pi^{2} \rho_{0} a r_{0}^{2} \int_{0}^{\infty} \delta\left(p-p_{0}\right) p^{2} \mathrm{~d} p \\
& N=\pi^{2} \rho_{0} a r_{0}{ }^{2} p_{0}{ }^{2}
\end{aligned}
$$$$
\text { and } \quad I=\pi^{2} e \rho_{0} a r_{0}{ }^{2} p_{0}{ }^{2}
$$

Since $r_{0}^{2} p_{0}^{2}$ has the dimensions of emittance, we replace it with the symbol $E$. The brightness $B$ of this very simple source is then given by the relationship :

$$
B=e \rho_{0} a=\frac{I}{\pi^{2} E^{2}}
$$

\section{References}

[1] Middleton R. and Adams C. T., University of Pennsylvania, Dept. of Physics Report (July 1973).

[2] Middleton R., University of Pennsylvania, Dept. of Physics Report (1977).

[3] Doucas G., Mck. Hyder H. R. and Knox A. B., Oxford University Nuclear Physics Laboratory Report 19 (1975).

[4] Brandt K., Nucl. Instrum. Methods 141 (1977) 519.

[5] Doucas G. and M(K. Hyder H. R., Nucl. Instrum. Methods 119 (1974) 413 and 124 (1975) 11.

[6] JuRela Z., Radiat Eff. 19 (1973) 175.
[7] J Ri La Z., Int. J. Mass Spectrom. Ion Phys. 18 (1975) 101.

[8] Paletto S., Goutte R. and Guillaud C., C. R. Hebd. Séan. Acad. Sci. 273 (1971) 975.

[9] Krohn V. E., J. Appl. Phys. 33 (1962) 3523.

[10] Mueller M. and Hortig G., IEEE Trans. Nucl. Sci. NS-16 (1969) 38.

[11] Simpson J. A., Rev. Sci. Instrum. 32 (1961) 1283.

[12] Doucas G., Int. J. Mass Spectrom. Ion Phys. (to be published). 